\title{
Using Agent-Based Modelling for Preliminary EER EXPERIMENTAL DESIGN
}

\author{
Robert W. Brennan*, Glenn Hermanson, Nancy Nelson, Robyn Paul, Monique Sullivan \\ Schulich School of Engineering, University of Calgary \\ *rbrennan@ucalgary.ca
}

\begin{abstract}
In this paper, we explore the use of agentbased modelling for preliminary engineering education research design. The motivation for this work is to help researchers understand the behaviour of the system being studied (e.g., classroom, program, etc.) before embarking on the, often, long process of an engineering education research study. We provide an example model of the effect of homework difficulty on homework completion and study group formation to demonstrate this approach. The results indicate this approach holds considerable promise as a preliminary modelling tool that can help focus longer-term engineering education research studies.
\end{abstract}

Keywords: Engineering Education Research Methods, Homework and Learning, Teamwork, Intention

\section{INTRODUCTION}

In recent years, there has been increasing interest in the use of agent-based modelling (ABM) as a tool to understand the behaviour of complex systems. This work has focused on the study of the dynamics of systems, such as organizations, whose behaviour is the consequence of the interactions of many different interdependent agents, such as individuals [21].

$\mathrm{ABM}$ is a form of computational modelling whereby a phenomenon is modelled by software agents and their interactions [27]. Early work in this area focused on understanding natural phenomena such as ant foraging, termite nesting, and bird flocking behaviour, as well as the behaviour of engineered systems such as freeways and computer networks [18]. In the past two decades interest has expanded to the study of social science systems [8], including studies of market economies, cooperation in groups, ethnocentrism, segregation, and language change [27].

As $\mathrm{Gu}$ and Blackmore [10] note in their systematic review of agent-based models, since 2009, this interest in $\mathrm{ABM}$ has expanded to higher education. Specific areas of interest for higher education include (1) studies of universities as independent organizational systems (e.g., [14] and [19]), (2) university collaboration (e.g., [1] and
[22]), (3) application and enrolment (e.g., [11] and [16]), (4) student performance (e.g., [4], [9], and [17]), and (5) teaching and learning (e.g., [13] and [15]). It should be noted that $\mathrm{Gu}$ and Blackmore's "teaching and learning" category relates to the actual teaching of ABM techniques rather than to the modelling of teaching and learning strategies. However, in recent years the study of alternative teaching and learning strategies using $\mathrm{ABM}$ has been approached by Seman et al. [20] - who investigate projectbased learning (PBL) with ABM - and Hostetler et al. [12] - who model student behaviour in the context of critical conversations.

In this paper, we explore the use of $\mathrm{ABM}$ as a preliminary modelling tool that can be used by engineering education researchers to inform their experimental designs. The motivation for this work is to help researchers understand the behaviour of the system being studied (e.g., classroom, program, etc.) before embarking on the, often, long process of an engineering education research (EER) study.

Most EER studies are studies of complex systems of the type described above: i.e., dynamic systems consisting of many heterogeneous, interacting agents (students, faculty, teaching assistants, etc.). These studies involve considerable uncertainty, and typically require sufficiently large sample sizes and timeframes to validate the effects of the study. As a result, there is always the risk that the time and effort put into the study can be wasted if errors are made in the early design phase of the study. To mitigate this risk, we propose performing preliminary $\mathrm{ABM}$ studies to understand the behaviour of the system. This will allow researchers to explore the behaviour of the system being studied, and in particular, its sensitivity to parameter changes. Ideally, these preliminary studies will inform the experimental design in a way that will result in welldesigned, and successful EER studies.

In this paper, we build upon the work on $A B M$ modelling of social science systems [8]. As a test-case, we focus on effect of graded homework on student learning. Our model uses the Theory of Planned Behaviour [2] as a framework for modelling student intention and behaviour with respect to homework completion. We develop an ABM to study the relationship between homework 
assignment and student learning [5,23]. When developing an ABM of a system such as this, it is helpful to start simple, then build complexity into the model. Thus for the purpose of this preliminary simplistic model, we assume learning is directly related to homework completion.

$\mathrm{ABM}$ is not intended to provide definitive, generalizable results for given EER studies. Instead, our intention is to provide an approach for preliminary, exploratory modelling that can inform the experimental design of EER studies. For example, the ABM will help researchers isolate important parameters from trivial parameters to better focus a study that may eventually involve many students and faculty over the course of one or more terms. Our hope is this approach will benefit the CEEA-ACEG community by offering an EER approach that saves time and helps focus one's research by providing high-level understanding of the research problem.

In the next section, we describe the agent-based model developed for this paper through the use of a simple test case. Next, we report on the results of this modelling approach in Section 3. It should be noted that less weight is placed on the results of the specific test case, as our focus is on the efficacy of the general modelling approach proposed in this paper. Finally, we offer our conclusions and suggestions for future study in Section 4.

\section{THE AGENT-BASED MODEL}

Agent-based modelling typically follows a three-stage process: constructing a conceptual model based on the relevant literature, developing the computational model using ABM software, and validation [10]. In order to explore the use of agent-based modelling in EER, we began by identifying a basic problem that could be used as a test case. For this paper, we chose the problem of homework assignment: i.e., whether or not homework should be assigned. In this section, we begin with a description of this test case (i.e., the conceptual model). We follow this with description of its agent-based model (i.e., the computational model) in Section 2.2, then a general discussion of the agent-based modelling approach (i.e., the approach to validation) in Section 2.3.

\subsection{The Test Case}

As Biggs and Tang [3] point out, "good teaching is that which supports the appropriate learning activities and discourages inappropriate ones". Given the orientation of many undergraduate engineering courses towards problem-solving, it is not surprising that many instructors view problem-solving practice as an appropriate learning activity for their courses. This view is also supported by the literature on the link between practice and proficiency $[5$, 28].

The most common learning activity associated with problem-solving practice is homework: in this paper, we use Cooper's [6] definition of homework as "any task assigned by [instructors] intended for students to carry out during non-school hours". However, questions often arise such as: How much homework should one assign? What grade weight should be assigned to the homework? What level of difficulty is appropriate?

The test case chosen for this paper is intended to address these types of questions by exploring the behaviour of students subjected to various homework conditions. The framework for student behaviour is based on Ajzen's [2] Theory of Planned Behaviour (TPB), which states that, even if an individual's attitude and subjective norm are in favour of committing a behaviour (e.g., completing homework), the individual might not be able to perform the behaviour. This framework is centred on individuals' intention: i.e., intention is a determinate of actual behaviour, and that intention is determined by attitude, subjective norm, and perceived behavioural control [2].

As will be discussed further in Section 2.3, we chose to start with a simple model where student intention is to complete homework assignments. For example, students' attitude towards homework is that it improves academic achievement, and the subjective norm is that other students share this attitude. Perceived behavioural control relates to the perceived ease or difficulty in performing a behaviour: in our test case, students are exposed to homework of varying levels of difficulty and adapt to this by forming study groups.

Our test case assumes students have a fixed "time budget" that can be used to complete homework with a given "time to complete". If a student's time budget is greater or equal to the time to complete for the homework, the student completes the homework. Otherwise, the student looks for other students who are in the same situation, and if successful, forms study groups with these students. When a study group has a combined time budget greater than or equal to the time to complete for the homework, the study group completes the homework. It should be noted that, in our model, we assume that students can only join study groups that have not completed the homework: in Section 4.1 we propose a model extension that would address academic integrity (e.g., sharing or copying homework solutions).

\subsection{The Agent-based Model}

The test case described previously is modelled using NetLogo 6.0.4 [25]. As a starting point for the model, we used elements from the Netlogo "Giant Component" model [26]. More specifically, we extended portions of this model's code for network exploration in order to manage the study groups in our model.

For the model, we have a single agent type, used to represent students. As noted previously, we chose to start with a simple agent model for the initial version of the ABM. As shown in Table 1, student agents are defined by three attributes and two behaviours. The classroom size is 
specified by the user (i.e., the number of student agent instances).

Table 1: Student agent characteristics.

\begin{tabular}{|l|ll|}
\hline Attributes: & $\begin{array}{l}\bullet \\
\end{array}$ & Time-Budget (time units) \\
& $\bullet$ & Completed? (True/False) \\
\hline Behaviours: & $\bullet$ & Complete Homework \\
& $\bullet$ & Join Study Group \\
\hline
\end{tabular}

Homework is modelled in the $\mathrm{ABM}$ as a set of environmental variables (i.e., global variables in the model). The user specifies the Time-to-Complete the homework ( 0 to 10 time units) and the Deadline (simulation run time) for the homework.

This is a stochastic model, where student Time-Budget is sampled from a uniform distribution (0 to 10 time units) for each student agent instance. It should be noted that the time units used in the model are not specified: i.e., they could take on any time unit such as seconds, minutes, days, etc. For the purposes of the model, we are interested in the relationship between Time-to-Complete and Time-Budget. As noted in Section 2.1, if a student has a Time-Budget greater than or equal to the homework Time-to-Complete, the homework is completed (i.e., the student agent's Completed? variable is set "true"). Similarly, if a study group has a combined Time-Budget greater than or equal to the homework Time-to-Complete, the homework is completed by all students in the study group.

The measures for the ABM fall in two categories: completion measures (number of students completed) and study group measures (number of study groups, study group size, number of students in study groups).

An example of an experimental run of the ABM is shown in Figure 1. Figure 1(a) shows the control panel where parameters such as the class size (num-nodes), Time-to-Complete, and Deadline (max-time) can be specified. As well, monitors and graphs of output measures are updated dynamically (e.g., Max Group Size, Ave Group Size, Students Completed). The bottom of the figure shows the status of each of the agents in the model (students are represented by simple "person" symbols). More specifically, agent colour represents the Completed? variable (green for true, grey for false), and lines between students show links to study groups.

The simulation run shown in Figure 1(b) also includes our first extension to the basic model described above. For this extension, we use two classes of student agents: (1) an extra-curricular student (shown by a larger person symbol) for students who are involved in extra-curricular activities (e.g., work, student clubs, varsity athletics, etc.), and (2) a regular student (shown by a smaller person symbol) for students who have no extra-curricular commitments. Each student class is further identified by the Extra? attribute: this attribute is "true" for students involved in extracurricular activities, and "false" for regular students. For this extension, the user can specify the proportion of students who are involved in extra-curricular activities (students-extra) and the impact of extra-curricular activities on these students' Time-Budget (impact-extra). In this case, $50 \%$ of the students are involved in extracurricular activities and these activities use of $50 \%$ of their Time-Budget.

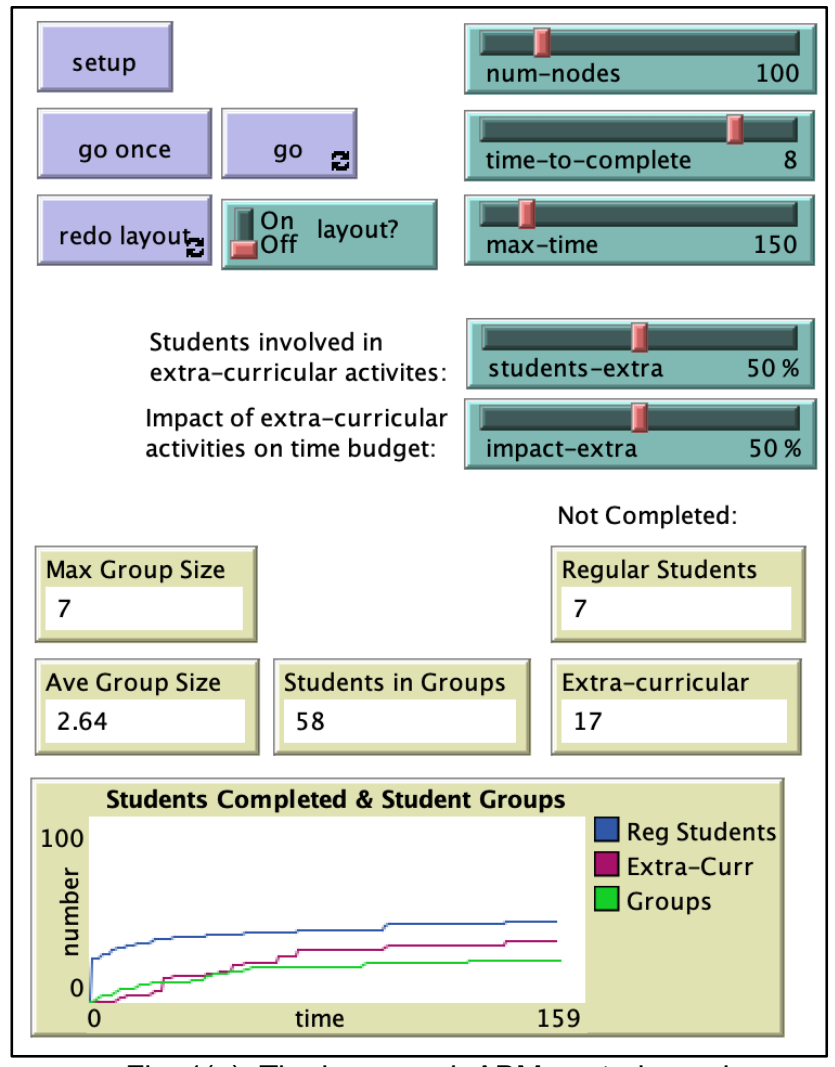

Fig. 1(a). The homework ABM control panel.

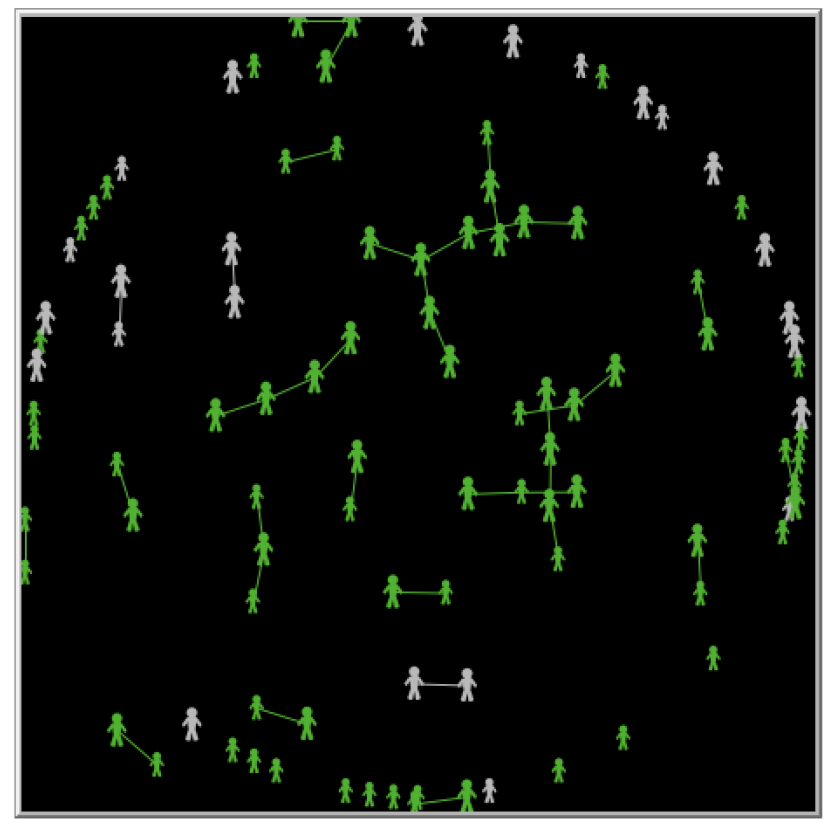

Fig. 1(b). The homework ABM simulation. 


\subsection{The Modelling Approach}

When developing an ABM of a system like the one described above, it is helpful to start simple, then build complexity into the model. In particular, it is useful to keep the agents as simple as possible with respect to the number of attributes and behaviours.

This is not just a matter of simplifying the modelling and coding process, but also a matter of isolating model parameters, then observing the effects on system behaviour before adding more complexity to the agents. This ability to incrementally build and experiment with the model, and to isolate parameters and observe effects is a major benefit of the ABM approach. This is difficult, if not impossible, to perform in a real life classroom setting.

As noted by Wilensky and Rand [27], it is also important to always be able to revert to the simpler, base case models whenever additional features are added to the ABM.

In our case, the "extra-curricular" extension can be reverted back to our base case by simply zeroing the students-extra and impact-extra selections shown in Figure 1(a). This ability to back-track aids the model verification process: i.e., if errors are found in extensions to the model, one can always revert to the last iteration, and move forward with model verification.

As with any modelling and simulation approach, validation is a key concern. The ABM presented in this paper is currently in its preliminary stage of development, and cannot be expected to provide an exact representation of the real world. However, at each stage of a model's development, it is important to observe how the model behaves with respect to parameter changes, and ask if the behaviour makes sense and can be explained. This process will support model validation by helping to build confidence in the ABM's output. Of course, the ultimate test of the model's validity occurs when the actual interventions are applied to the real system (e.g., in the classroom setting).

\section{RESULTS AND DISCUSSION}

For all experiments run with the Homework ABM, we varied the Time-to-Complete variable in five steps $(2,4,6$, 8 , and 10 time units) in order to observe the student behaviour for increasingly difficult homework assignments. More specifically, we collected data on homework completion (number of students who did not complete the homework) and study group formation (number of study groups, number of students in study groups, and average study group size) for each increase in the Time-to-Complete variable.

We performed 10 simulation runs for each parameter change, which allowed us to collect typical statistical measures for each performance measure. Given that each simulation run takes less than 10 seconds to perform, it would be straight-forward to increase the number of replications if needed.

For the first iteration of the model (i.e., no students involved in extra-curricular activities), we found that most students completed the homework, and as expected, this number decreased as homework difficulty (Time-toComplete) increased: i.e., the number of students who completed the homework ranged from 92\% (2 time unit homework) to $88 \%$ (10 time unit homework). The relatively small difference in this measure is most likely due to our choice of a very long Deadline for this set of experiments (400 time units).

As shown by the box plots in Figures 2 (below) and Figure 3 (on the next page), the ABM showed some interesting behaviour for the study group metrics. As homework difficulty increases, the number of students participating in study groups increases significantly (Figure 2). However, the average study group size remained approximately the same (2 to 3 students) regardless of homework difficulty (Figure 3 ).

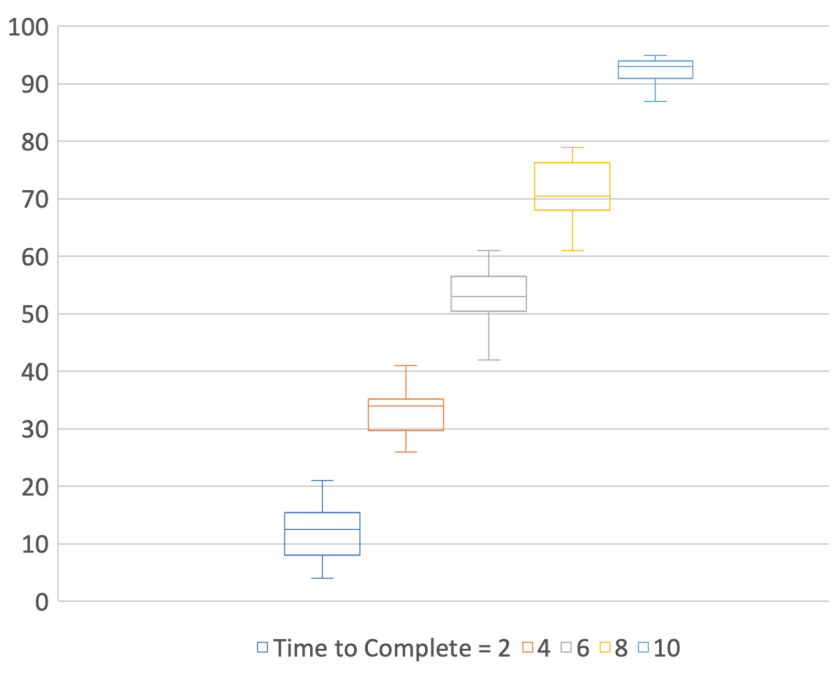

Fig. 2. Number of students participating in study groups for increasing homework difficulty (time-to-complete).

These results suggest that the effect of homework difficulty on study group formation could be an interesting area for further exploration. For example, an instructor's choices around the level of difficulty of homework assignments could influence individual vs. team work behaviour. As well, it would be interesting to explore if the average group size results are telling us anything about ideal group sizes.

The next iteration of the ABM recognizes that many undergraduate engineering students are involved in extracurricular activities that can negatively impact time available for curricular activities (such as homework). For the experiments, we also shortened the Deadline from 400 time units to 150 time units in order to get a clearer picture 
of how homework difficulty impacts homework completion.

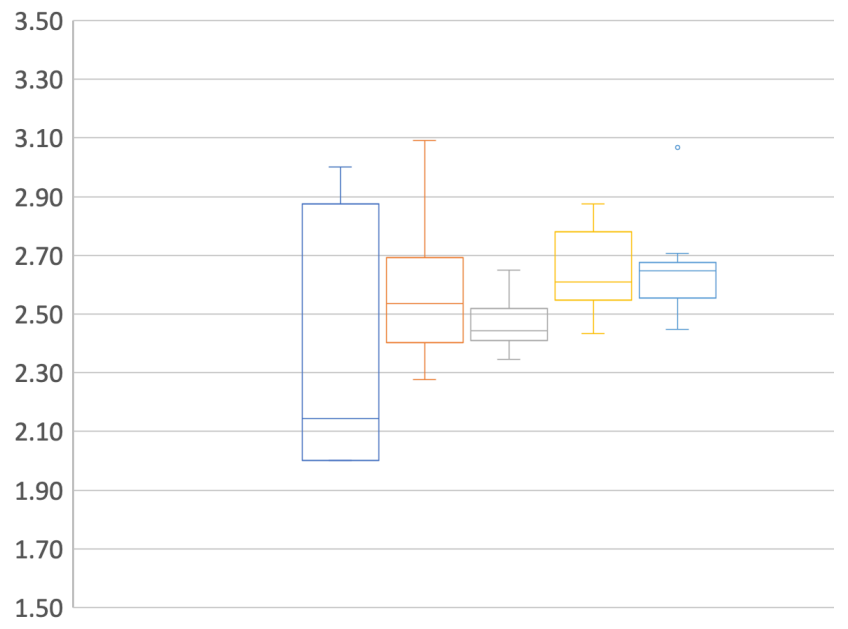

$\square$ Time to Complete $=2 \square 4 \square 6 \square 8 \square 10$

Fig. 3. Average study group size for increasing homework difficulty (time-to-complete).

The homework completion results are shown in Figure 4. This figure shows box plots of the number of students who did not complete the homework by the 150 time unit Deadline. Both the "regular" students (highlighted with the blue line through the means) and the "extra-curricular" students (highlighted with the red line through the means) are shown.

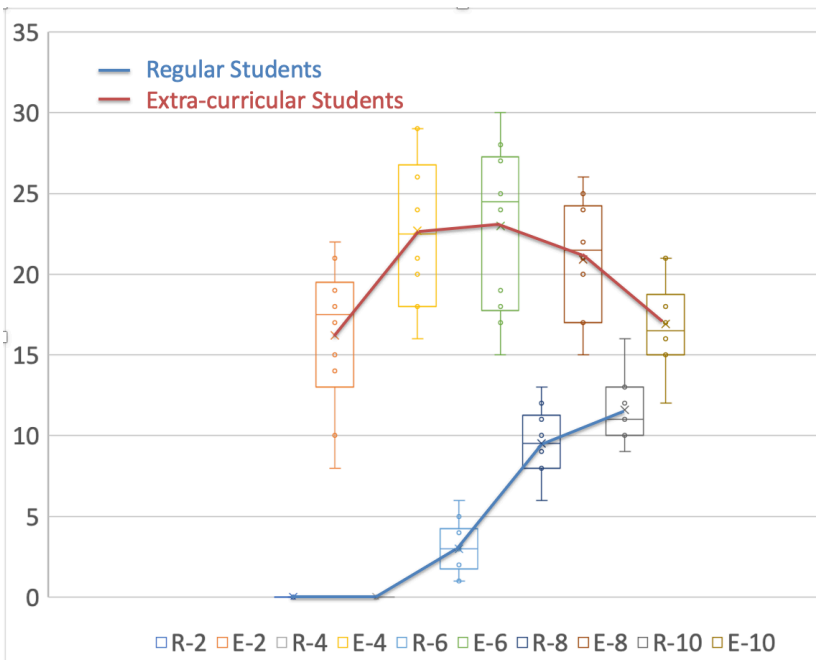

Fig. 4. Number of students who did not complete the homework by the deadline (150 time units).

As expected, the group of students who are not involved in extra-curricular activities (and have more time budget for homework) have lower numbers of students who have not completed the homework than those involved in extra- curricular activities. As well, increases in homework difficulty result in more students not completing the homework. However, this measure increases, then decreases for students involved in extra-curricular activities.

At first glance, this result may appear counter-intuitive. However, Figure 4 is showing a phenomenon that is a result of the overall interactions of heterogeneous agents (i.e., "regular" and "extra-curricular" students). The number of study groups and the number of students participating in study groups increase as the homework difficulty increases (cf. Figures 2 and 3). As the homework difficulty increases, regular and extra-curricular students are becoming more alike: i.e., both groups need to join study groups to get the homework done. As a result, we see an increase in the number of study groups and the number of students participating in study groups, and a corresponding increase in opportunities to join study groups (for both types of student). This results in the convergence of the two student types shown in Figure 4.

Given the increased focus on student clubs and teams in many undergraduate engineering programs, as well as increasing pressure for students to take on part-time work to subsidize their education, it would be interesting to explore this relationship further.

\section{CONCLUSIONS AND FUTURE WORK}

As noted previously, the primary motivation for the work presented in this paper was to explore whether or not agent-based modelling could serve as a useful tool for preliminary EER experimental design. Based on our experience with the Homework ABM, we feel the answer is "yes": this approach has considerable potential and is certainly worth pursuing for a broad range of EER studies.

For example, for this particular test case, we developed a simple model of student behaviour when confronted with increasingly more difficult homework assignments. To replicate an experiment like this in the classroom, it would involve more than 100 students (assuming some students would opt out of the study), and an intervention involving 5 different types of homework assignments. To perform all of the interventions, the researchers would require at least one term (12 weeks) to perform the study.

Although this type of study is certainly feasible, there would be very little (if any) latitude for change given research ethics stipulations and the practicalities of running the study during an academic term. For example, changes of the type noted for our test case (adjustment of the homework deadline or asking students about their involvement in extra-curricular activities) would typically require a modification to the research ethics proposal, and possibly a re-run of the experiment.

In addition to providing flexibility to perform exploratory studies, the ABM approach also provides researchers with opportunities to break through pre- 
conceived notions and/or biases [24], and potentially, open up new directions for the EER study. For example, we did not anticipate the homework completion behaviour illustrated in Figure 4. We hypothesized this behaviour is a consequence of the study group formation behaviour described previously: this would be interesting to verify through further studies with the ABM, and ultimately, in the classroom.

In the remainder of this section, we offer our thoughts on future work in the area. We begin with possible extensions to the Homework ABM model, then conclude with possible next steps for ABM modelling as an EER preliminary experimental design tool.

\subsection{The Homework ABM}

In the current $\mathrm{ABM}$, the student agents have a very simple set of attributes and behaviours (Table 1). As noted in Section 2.3, it was our intention to start simple, then build complexity into the model as part of our general modelling approach. As such, the extensions to the Homework ABM discussed in this section are of this nature.

One potentially promising extension relates to "homework completion efficiency". Currently, all student agents use whatever time budget is available to them in the same way to complete the homework. However, real students have varying degrees of studying efficiency: i.e., some are more efficient at completing homework than others for a variety of reasons (e.g., better study skills, higher motivation, less distractions, etc.). A homework completion efficiency factor may also have a relationship with the student types described earlier. For example, students involved in extra-curricular activities are busy and recognize they must be efficient with their time.

A second area of interest is that of academic integrity. As noted previously, once a study group has completed a homework assignment, it does not accept additional students. However, there may be cases where study groups or study group members are willing to share solutions (and students are willing to use these solutions to meet a homework deadline). Similarly, there may be situations where a solution is available that can be used by students who wish to take short-cuts (e.g., a solution manual, a completed homework from previous years, etc.). This can be thought of as a variation of the scenario of joining "completed" study groups. For this extension, we are interested in expanding on the Theory of Planned Behaviour framework described previously. More specifically, we would use Cronan et al.'s [7] work as a starting point for modelling student agent intentions with respect to academic integrity.

\subsection{Agent-based Modelling for EER}

The notion of building an agent-based model from scratch can be daunting for researchers who have not had experience with this approach. Even if $\mathrm{ABM}$ looks promising and beneficial, the learning curve and the time to develop the model pose barriers to many researchers. To address this problem and to help EER researchers interested in $\mathrm{ABM}$, we are currently developing a general design process for ABM in EER: the preliminary outline of this approach is discussed in Section 2.3.

However, a general design process for ABM will not be enough: resources are required to reduce the time and effort to develop agent-based models. In this regard, we recommend the CEEA-ACEG community uses a common $\mathrm{ABM}$ platform, and that interested researchers contribute to a shared repository of base models for EER researchers.

Based on our experience, we found Netlogo 6.0.4 [25] to be a good choice for a common ABM platform. It is available as a free download on all common operating systems, it is well-supported, is stable, and it already has a very large model library that can be built upon.

As discussed in Section 2.2, the Homework ABM described in this paper used Wilensky's "Giant Component" Netlogo model [26] as a starting point. The resulting model allowed us to explore student interactions and study group formation. In terms of our proposal for a shared repository of base models for EER researchers, the Homework ABM could be considered as a base case for explorations of student teams. For example, the Homework $\mathrm{ABM}$ could be expanded to look at questions around optimum team size in capstone design projects, the effect of different student team formation strategies (e.g., assigned v. self-selected), "loafing" behaviour in teams and study groups, etc.

Similarly, our proposed extension to explore academic integrity with the Homework ABM could result in a base model for studying a variety of research questions that centre on student intentions within the Theory of Planned Behaviour framework.

The general idea behind developing a shared repository of base models for EER researchers is that these models could serve as the starting point for this general ABM approach, just as the Giant Component model served as a starting point for the Homework ABM. Other examples of base models could include network models (to explore curriculum interactions), competition models (to explore student competitions, academic integrity, etc.), propagation models (to explore the propagation of beliefs, solutions, etc.), and segregation models (to explore group formation, curriculum interactions, etc.).

At the beginning of this paper we expressed our hope that this approach will benefit the CEEA-ACEG community by offering an EER approach to save time and to help focus one's research by providing high-level understanding of the research problem. It is our belief that ABM can provide these benefits; however, the greatest benefit will come from a shared approach involving a common platform and shared base models. 


\section{Acknowledgements}

The authors wish to thank the Natural Sciences and Engineering Research Council, Spartan Controls, the Suncor Energy Foundation, and the Schulich School of Engineering for their generous support of this research.

\section{References}

[1] Petra Ahrweiler, Andreas Pyka, and Nigel Gilbert. "A new model for university-industry links in knowledgebased economies," Journal of Product Innovation Management, vol. 28, no. 2, pp. 218-235, 2011.

[2] Icek Ajzen, "From intentions to behavior: a theory of planned behavior," in Action-Control: From Cognition to Behavior, J. Kuhl and J. Beckman (eds), Springer, Heidelberg, pp. 11-39, 1985.

[3] John Biggs and Catherine Tang, Teaching for Quality Learning at University, Fourth Edition, McGraw Hill, 2011.

[4] Yuzhuo Cai, "Graduate employability: a conceptual framework for understanding employers' perceptions," International Journal of Higher Education and Educational Planning, vol. 65, no. 4, pp. 457-69, 2013.

[5] Harris Cooper, Homework, White Plains, NY: Longman, 1989.

[6] Harris Cooper, Jorgianne C. Robinson, and Erika A. Patall, "Does homework improve academic achievement? A synthesis of research, 1987-2003," Review of Educational Research, vol. 7, no. 1, pp. 1-62, 2006.

[7] Timothy P. Cronan, Jeffrey K. Mullins, and David E. Douglas, "Further understanding factors that explain freshman business students' academic integrity intention and behavior: plagiarism and sharing homework," Journal of Business Ethics, vol. 147, pp. 197-220, 2018.

[8] Joshua Epstein, Generative Social Science: Studies in Agent-computational Modeling, Princeton University Press, Princeton, 2006.

[9] Luca Gerotto, and Paolo Pellizzari, "Unemployment expectations in an agent-based model with education," Lecture Notes in Computer Science, vol. 10978, pp. 17586, 2018.

[10] X. Gu and K.L. Blackmore, "A systematic review of agent-based modelling and simulation applications in the higher education domain," Higher Education Research and Development, vol. 34, no. 5, pp. 883-98, 2015.

[11] Leslie Henrickson, "Old wine in a new wineskin: college choice, college access using agent-based modeling," Social Science Computer Review, vol. 20, no. 4, pp. 40019, 2002.

[12] Andrew Hostetler, Pratim Sengupta, and Ty Hollett, "Unsilencing critical conversations in social-studies teacher education using agent-based modeling,"
Cognition and Instruction, vol. 36, no. 2, pp. 139-70, 2018.

[13] A. Kurkovsky, "Interdisciplinary systems and simulation studies for an innovative undergraduate program," in Proceedings of the Emerging M\&S Applications in Industry \& Academia - Modeling and Humanities Symposium, 2013.

[14] Daniel Little, "Guiding and modelling quality improvement in higher education institutions," Quality in Higher Education, vol. 21, no. 3, pp. 312-27, 2015.

[15] C. Macal and M. North, "Successful approaches for teaching agent-based simulation," Journal of Simulation, vol. 7, no. 1, pp. 1-11, 2013.

[16] V. Moskovkin, "Simulation expert system for making students' college decisions," Automatic Documentation and Mathematical Linguistics, vol. 43, no. 5, pp. 292-95, 2009.

[17] Sean Reardon, Matt Kasman, Daniel Klasik, and Rachel Baker, "Agent-based simulation models of the college sorting process," Journal of Artificial Societies and Social Simulation, vol. 19, no. 1, p. 1, 2016.

[18] Mitchel Resnick, Turtles, Termites, and Traffic Jams: Explorations in Massively Parallel Microworlds, The MIT Press, Cambridge, MA, 1997.

[19] Paul J. Roebber and Richard G. Meadows, "Simulating alternative approaches to addressing fiscal resource tensions and quality in U.S. public higher education," Journal of Education Finance, vol. 38, no. 1, pp. 81-109, 2012.

[20] Laio Oriel Seman, Romeu Hausmann and Eduardo Augusto Bezerra, "Agent-based simulation of learning dissemination in a project-based learning context considering the human aspects," IEEE Transactions on Education, vol. 61, no. 2, pp. 101-08, 2018.

[21] Transformative Learning Technologies Lab, Stanford Graduate School of Education, Available as of 13 January 2019 from https://tltl.stanford.edu.

[22] Giorgio Triulzi and Andreas Pyka, "Learning by modeling: insights from an agent-based model of university-industry relationships," Cybernetics and Systems, vol. 42, no. 7, pp. 484-501, 2011.

[23] H. Joel Trussell and E. Jacquelin Dietz, "A study of the effect of graded homework in a preparatory math course for electrical engineers", Journal of Engineering Education, vol. 92, no. 2, pp. 141-146, 2003.

[24] Shankar Vdantam, Camila Vargas-Restrepo, Tara Boyle, Jennifer Schmit, "How science spreads: smallpox, stomach ulcers, and "the vegetable lamb of Tartary", Hidden Brain Podcast, National Public Radio, 21 January 2019, Available as of 17 February 2019 from https://www.npr.org/2019/01/11/684435633/howscience-spreads-smallpox-stomach-ulcers-and-thevegetable-lamb-of-tartary.

[25] Uri Wilensky, NetLogo, Center for Connected Learning and Computer-Based Modeling, Northwestern 
University, Evanston, IL, 1999, Available as of 17 February 2019 from http://ccl.northwestern.edu/ netlogo/.

[26] Uri Wilensky, NetLogo Giant Component model, 2005, Center for Connected Learning and Computer-Based Modeling, Northwestern University, Evanston, IL, Available as of 17 February 2019 from http://ccl.northwestern.edu/netlogo/models/GiantCompo nent
[27] Uri Wilensky and William Rand, An Introduction to Agent-based Modeling, The MIT Press, Cambridge, MA, 2015.

[28] Daniel T. Willingham, Why Don't Students Like School?: A Cognitive Scientist Answers Questions About How the Mind Works and What It Means for the Classroom, Jossey-Bass; 1st (first) edition, 2010. 